\title{
Median Mental Sinus in Twins
}

\begin{abstract}
Sinus on the chin can be the result of a chronic apical abscess due to pulp necrosis of a mandibular anterior tooth. The tooth is usually asymptomatic, and a dental cause is therefore not apparent to the patient or the unsuspecting clinician. Not infrequently, the patient may seek treatment from a dermatologist or general surgeon instead of a dentist. Excision and repair of the fistula may be carried out with subsequent breakdown because the dental pathology is not removed. This paper reports the presence of median mental sinus of dental origin in twins. One case healed following root canal therapy while the other required both root canal therapy and surgery to eliminate the infection.
\end{abstract}

\begin{tabular}{|l|l|}
\hline Authors: & Ong, S. T.; Ngeow, W.C. \\
\hline Journal: & Dental update \\
\hline Year: & 1999 \\
\hline Pages: & $163-165$ \\
\hline
\end{tabular}

\section{Keywords :}

Adolescent; article; case report; chin; endodontics; female; human; periodontitis; skin; fistula; tooth fistula; twins; Cutaneous Fistula; Dental Fistula; Diseases in Twins; Humans; Periodontal Abscess; Root Canal Therapy

\section{Please cite as :}

ONG, S. T. \& NGEOW, W. C. 1999. Median mental sinus in twins. Dental update, $26,163-165$. 


\section{URL :}

- http://www.scopus.com/inward/record.url?eid=2-s2.00033123059\&partnerID $=40 \& m d 5=810 \mathrm{a} 5 \mathrm{~d} 49 \mathrm{~b} 6 \mathrm{bb} 9412 \mathrm{f} 435 \mathrm{fa} 01 \mathrm{f} 71 \mathrm{f} 625 \mathrm{c}$

- http://www.ncbi.nlm.nih.gov/pubmed/10765768

- $\quad$ http://lib.bioinfo.pl/pmid:10765768

- http://www.researchgate.net/publication/12550315_Median_mental_sinus_in_twins

- http://ukpmc.ac.uk/abstract/MED/10765768 